\title{
Autosomal dominant pure spastic paraplegia: a clinical, paraclinical, and genetic study
}

\author{
J E Nielsen, K Krabbe, P Jennum, P Koefoed, L Neerup Jensen, K Fenger, H Eiberg, \\ L Hasholt, L Werdelin, S A Sørensen
}

Institute of Medical Biochemistry and Genetics, Laboratory of Medical Genetics, Section of

Neurogenetics, University of

Copenhagen, Denmark

$\mathrm{J}$ E Nielsen

P Koefoed

K Fenger

H Eiberg

L Hasholt

S A Sørensen

University Clinic of Neurology, Hvidovre Hospital, Copenhagen, Denmark

J E Nielsen

Danish Research Center of Magnetic Resonance, Hvidovre Hospital, Denmark K Krabbe

Department of Clinical Neurophysiology, Hvidovre Hospital, Copenhagen, Denmark $P$ Jennum

University Clinic of Urology, Herlev Hospital, Denmark

L Neerup Jensen

University Clinic of Neurology,

Frederiksberg

Hospital, Denmark

L Werdelin

Correspondence to: Dr J E Nielsen, Institute of Medical Biochemistry and Genetics, Laboratory of Medical Genetics, Section of Neurogenetics, The Panum Institute, Building 24.4, Blegdamsvej 3, DK-2200 Copenhagen. N Denmark. Telephone 00453532 7816; fax 00453139 3373; email: jnielsen@medgen.imbg.ku.dk

Received 2 September 1996 and in final revised form 17 June 1997

Accepted 30 June 1997

\begin{abstract}
Objectives-At least three clinically indistinguishable but genetically different types of autosomal dominant pure spastic paraplegia (ADPSP) have been described. In this study the clinical, genetic, neurophysiological, and MRI characteristics of ADPSP were investigated.

Methods-Sixty three at risk members from five families were clinically evaluated. A diagnostic index was constructed for the study. Microsatellite genotypes were determined for chromosomes $2 p$, $14 q$, and $15 q$ markers and multipoint linkage analyses were performed. Central motor conduction time studies (CMCT), somatosensory evoked potential (SSEP) measurement, and MRI of the brain and the total spinal cord were carried out in 16 patients from four families.
\end{abstract}

Results-The clinical core features of ADPSP were homogeneously expressed in all patients but some features were only found in some families and not in all the patients within the family. In two families non-progressive "congenital" ADPSP was seen in some affected members whereas adult onset progressive ADPSP was present in other affected family members. As a late symptom not previously described low backache was reported by $47 \%$. Age at onset varied widely and there was a tendency for it to decline in successive generations in the families, suggesting anticipation. Genetic linkage analysis confined the ADPSP locus to chromosome 2p21-p24 in the five families. The lod scores obtained by multipoint linkage analysis were positive with a combined maximum lod score of $Z=8.60$. The neurophysiological studies only showed minor and insignificant prolongation of the central motor conduction time and further that peripheral conduction and integrity of the dorsal columns were mostly normal. Brain and the total spinal cord MRI did not disclose any significant abnormalities compared with controls.

Conclusions-ADPSP linked to chromosome 2 p21-p24 is a phenotypic heterogeneous disorder characterised by both interfamilial and intrafamilial variation. In some families the disease may be "pure" but the existence of "pure plus" families is suggested in others. The neurophysiological and neuroimaging investigations did not show any major abnormalities.

(F Neurol Neurosurg Psychiatry 1998;64:61-66)
Keywords: autosomal dominant pure spastic paraplegia linked to chromosome $2 \mathrm{p}$; clinical features; neurophysiology; magnetic resonance imaging

Hereditary spastic paraplegias comprise a heterogeneous group of rare neurodegenerative disorders. Conventionally they are divided into two groups, depending on whether the disorder is a pure spastic paraplegia or a more complex syndrome with other associated features. ${ }^{1}$ Autosomal dominant pure spastic paraplegia (ADPSP) is clinically characterised by slowly progressive spasticity and weakness of the legs, hyperreflexia, and Babinski's sign, with little or no involvement of the upper limbs; the phenotypical expression of ADPSP being highly variable, particularly with respect to age at onset. By linkage analyses ADPSP has been mapped to the chromosomes 14q (the SPG3 locus), $2 \mathrm{p}$ (SPG4), and $15 \mathrm{q}$ (SPG6). ${ }^{2-4}$ As ADPSP in some families does not map to any of these loci additional, still unidentified genes may exist. ${ }^{5}$

The neuropathological findings are almost exclusively confined to the spinal cord and include degeneration of the lateral corticospinal tracts decreasing from the lower lumbar to the upper cervical level. Often, involvement of the uncrossed pyramidal tracts and increasing degeneration of the fasciculus gracilis from the lumbar to upper cervical level is also described. ${ }^{6}$ These findings are in part reflected in the paraclinical findings using transcranial magnetic or electrical stimulation of the motor cortex, somatosensory evoked potentials (SSEPs), and nerve conduction studies. $^{7-9}$ Although MRI provides superior images of the brainstem, cerebellum, and spinal cord, only small series of patients with ADPSP have been studied. ${ }^{10}{ }^{11}$ The purpose of this paper is to present the clinical, paraclinical, and genetic features in each of five families with ADPSP, and to discuss the interfamilial and intrafamilial variation.

\section{Patients and methods}

PATIENTS

Probands were searched for in the records from the neurological outpatient clinic at The Hvidovre Hospital and at the Institute of Medical Genetics in Copenhagen. After informed consent, family members were seen at home or in hospital. The diagnosis was made on the basis of a well documented family history and the diagnostic criteria of Harding ${ }^{1}$ and Bruyn and Scheltens. ${ }^{12}$ Minimal criteria for diagnosis were spasticity of the lower limbs, usually more pronounced than weakness, hyperactive tendon reflexes, and Babinski's 
Table 1 Diagnostic index for ADPSP

\begin{tabular}{ll}
\hline ADPSP 3 & $\begin{array}{l}\text { All four obligatory signs are found at } \\
\text { examination }\end{array}$ \\
ADPSP 2 & Three of the obligatory signs are found \\
ADPSP 1 & Two of the obligatory signs are found \\
ADPSP 0-1 & One of the obligatory signs is found \\
ADPSP 0 & No symptoms or signs of ADPSP \\
\hline
\end{tabular}

sign. A diagnostic index was constructed for the study (table 1).

Sixty three persons from five families (designated A-E) were personally examined, except for three persons from family A (IV-10, V-7, $\mathrm{V}-8$, fig 1), who live in Sweden; all three, however, were reported by several family members to be affected and their history was confirmed by telephone contact. Persons who were dead were designated as affected if they were said to have had "the family disease" by more than one family member or by hospital records. Blood samples were obtained for isolation of DNA.

A three point functional grading scale, modified from Behan and Maia, was adopted for the study. ${ }^{13}$ Grade 1 corresponds to an asymptomatic patient with pyramidal signs in the lower limbs with normal or only slightly spastic gait. Grade 2 refers to a patient with spastic gait, able to walk independently with or without support. Grade 3 refers to a chairbound or bedridden patient.

Five patients from family $\mathrm{A}$, four patients from family B, four patients from family C, and three patients from family $\mathrm{D}$, ranging from grade 1 to 3 underwent transcranial magnetic stimulation, SSEP studies, and MRI of the brain and spinal cord. For geographical reasons patients from family $\mathrm{E}$ were not available for the paraclinical studies.

GENETIC LINKAGE STUDIES

Genomic DNA was extracted from leucocytes using standard procedures. Microsatellite genotypes were determined for the SPG3, SPG4, and SPG6 loci. One of each primer pair
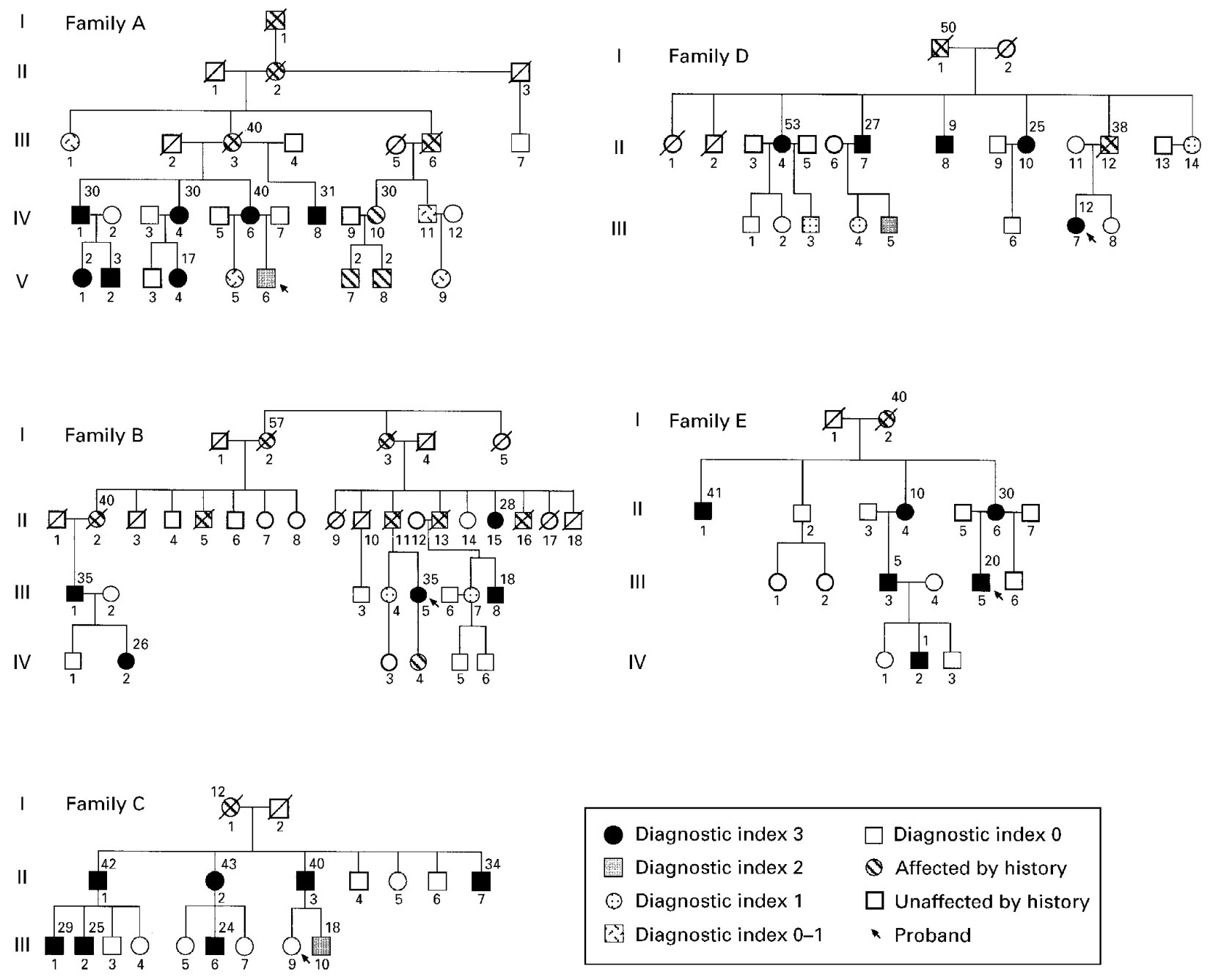

\begin{tabular}{ll}
\hline Diagnostic index 3 & $\square$ Diagnostic index 0 \\
Diagnostic index 2 & $\bigcirc$ Affected by history \\
$\odot$ Diagnostic index 1 & $\square$ Unaffected by history \\
Diagnostic index $0-1$ & $\times$ Proband
\end{tabular}

Figure 1 Pedigrees of the five families ( $A-E)$ with ADPSP. Numbers above symbols indicate age at onset. 
Table 2 Diagnostic index, age at onset, disease duration, and disability score (median, range) in five families with ADPSP

\begin{tabular}{|c|c|c|c|c|c|c|}
\hline Family & $A$ & $B$ & $C$ & $D$ & $E$ & No \\
\hline Examined (n) & 16 & 11 & 15 & 12 & 9 & 63 \\
\hline Diagnostic index 3 & $7(+3)$ & 5 & 7 & 5 & 6 & $30(+3)$ \\
\hline Diagnostic index 2 & 1 & 0 & 1 & 1 & 0 & 3 \\
\hline Diagnostic index 1 & 0 & 2 & 0 & 3 & 0 & 5 \\
\hline Diagnostic index 0-1 & 4 & 0 & 0 & 0 & 0 & 4 \\
\hline Diagnostic index 0 & 1 & 4 & 7 & 3 & 3 & 18 \\
\hline No symptoms but signs & 3 & 2 & 0 & 4 & 0 & 9 \\
\hline No diagnostic index 3 & 7 & 5 & 7 & 5 & 6 & 30 \\
\hline Age at onset & $30(2-40)$ & $28(18-35)$ & $34(24-43)$ & $25(9-53)$ & $15(1-41)$ & \\
\hline Duration & $24(13-33)$ & $25(3-50)$ & $9(5-14)$ & $24(3-42)$ & $17.5(4-43)$ & \\
\hline Disability score & $2(1-2)$ & $2(2-3)$ & $2(2-3)$ & $2(2-2)$ & $2(2-3)$ & \\
\hline
\end{tabular}

(+3) Refers to the three affected people living in Sweden.

was end labelled, using $\left(\gamma-{ }^{32} \mathrm{P}\right) \mathrm{ATP}$ and $\mathrm{T} 4$ polynucleotide kinase (Pharmacia Biotech).

Polymerase chain reaction (PCR) was carried out in a Perkin Elmer GeneAmp PCR system 2400 using conditions as given by Bürger et $a l^{14}$ with minor modifications: Annealing temperatures were $59^{\circ} \mathrm{C}$ for $\mathrm{D} 2 \mathrm{~S} 400 ; 57^{\circ} \mathrm{C}$ for D14S266, D14S269, and D15S156; $55^{\circ} \mathrm{C}$ for D15S128 and D15S986.

The lod scores were calculated by multipoint analysis using the LINKMAP program from the FASTLINK package ${ }^{15}$ with markers positioned in accordance with the Généthon map. ${ }^{16}$ The markers and distances (cM) used for the multipoint analysis were chromosome 2: D2S400-(0.0)-D2S352-(0.01)D2S2351 - (0.01) - D2S2374 - (0.0) - D2S367; chromosome 14: D14S266-(6.0)-D14S269; chromosome 15: D15S128-(8.0)-D15S156.

\section{ELECTROPHYSIOLOGICAL TECHNIQUES}

Central motor conduction time (CMCT) measurements were performed on the right and left abductor pollicis brevis, biceps brachii, vastus medialis, anterior tibial, extensor digitorum brevis, and abductor hallucis muscles. The active electrode was placed over the midpart of the muscle, the reference over the tendon distal to the recording electrode. The motor threshold was defined as the lowest stimulus intensity that gave a reproducible magnetic evoked potential (MEP) with an amplitude exceeding $50 \mu \mathrm{V}$ in five consecutive stimuli. The stimulations were applied to the contralateral cortical area representing the muscle, as defined by the

Table 3 Symptoms and signs in 30 affected persons (ADPSP index 3) in the five families

\begin{tabular}{|c|c|c|c|c|c|}
\hline Family & $A$ & $B$ & $C$ & $D$ & $E$ \\
\hline No & 7 & 5 & 7 & 5 & 6 \\
\hline \multicolumn{6}{|l|}{ Symptoms at onset: } \\
\hline Delayed motor milestones & 2 & 0 & 0 & 0 & 1 \\
\hline Gait disturbance & 6 & 4 & 6 & 4 & 6 \\
\hline Stiffness of lower limbs & 5 & 5 & 7 & 5 & 6 \\
\hline Poor balance & 6 & 1 & 4 & 1 & 4 \\
\hline Problems going down stairs & 4 & 4 & 6 & 3 & 4 \\
\hline Rapidly worn out shoes & 6 & 5 & 5 & 5 & 5 \\
\hline Poor athletic performance & 1 & 0 & 5 & 2 & 4 \\
\hline Low backache & 4 & 3 & 3 & 2 & 2 \\
\hline \multicolumn{6}{|l|}{ Signs (UL LL): } \\
\hline Weakness & $0 / 77 / 7$ & $1 / 55 / 5$ & $1 / 77 / 7$ & $0 / 55 / 5$ & $1 / 65 / 6$ \\
\hline Spasticity & $0 / 77 / 7$ & $2 / 55 / 5$ & $2 / 77 / 7$ & $1 / 55 / 5$ & $1 / 66 / 6$ \\
\hline Hyperreflexia & $4 / 77 / 7$ & $5 / 55 / 5$ & $2 / 77 / 7$ & $3 / 55 / 5$ & $5 / 66 / 6$ \\
\hline Amyotrophy & $0 / 74 / 7$ & $0 / 52 / 4$ & $0 / 72 / 7$ & $1 / 52 / 5$ & $1 / 63 / 6$ \\
\hline Vibration sense decreased & $0 / 72 / 7$ & $1 / 51 / 5$ & $0 / 74 / 7$ & $0 / 53 / 5$ & $0 / 52 / 5$ \\
\hline Dysdiadochokinesia & $1 / 71 / 7$ & $0 / 50 / 5$ & $3 / 70 / 7$ & $0 / 50 / 3$ & $3 / 61 / 5$ \\
\hline Nystagmus (horizontal) & $3 / 7$ & $1 / 5$ & $3 / 7$ & $0 / 5$ & $0 / 6$ \\
\hline Dysarthria & $0 / 7$ & $0 / 5$ & $1 / 7$ & $0 / 5$ & $2 / 6$ \\
\hline Urinary symptoms & $4 / 7$ & $0 / 5$ & $5 / 7$ & $0 / 5$ & $4 / 6$ \\
\hline Pes cavus & $7 / 7$ & $5 / 5$ & $4 / 7$ & $4 / 5$ & $4 / 6$ \\
\hline
\end{tabular}

$\mathrm{UL}=$ upper limbs; $\mathrm{LL}=$ lower limbs. area with the lowest motor threshold. All stimulations were applied with relaxed (nonfacilitated) muscles. The stimulus intensity was $1.5 \times$ motor threshold. Cervical and lumbar stimulation were applied. The total and the cervicolumbar latencies were measured and the CMCTs were calculated.

The SSEPs were obtained from stimulation of the left and right median nerves at the wrist and the tibial nerves at the medial malleolus. The SSEPs from the median nerves were obtained from Erbs point, C7, and from the contralateral postcentral region. The SSEPs from the tibial nerves were recorded from the gluteal region, Th12, and from the postvertex region. The stimulus intensity was about $50 \%$ above the threshold for a visible contraction of the abductor pollicis brevis and abductor hallucis muscles. A detailed report of the method and normative data were presented by Pedersen and Trojaborg. ${ }^{17}$ The results from all measurements were compared with the results from healthy controls with similar age and height distributions.

MAGNETIC RESONANCE IMAGING

Brain and spinal cord MRI were performed in a 1.0 Tesla superconducting system (Siemens Impact). The brain was imaged using axial and coronal double spin echo sequences (2200/ 20/80 ms (repetition time (TR)/echo time (TE)/echo time (TE))), and sagittal T1 weighted spin echo images (570/15 (TR/TE)). In the spinal cord sagittal double spin echo images (2200/20/80 ms (TR/TE/TE)) and T1 weighted images (500/15 (TR/TE)) were obtained. Similar MRI was performed in healthy controls matched for age and sex. Two experienced radiologists evaluated the images of patients and controls and reported by consensus for signs of pathology. The cerebrum, cerebellum, brainstem, and medulla oblongata were evaluated for the presence of atrophy on a three point grading scale (0: no atrophy; 1: mild atrophy; 2: severe atrophy). Hyperintense lesions on T2 weighted images were counted and any other sign of pathology was noted. The spinal cord was evaluated for the presence of atrophy on $\mathrm{T} 1$ weighted images and examined for hyperintense lesions on T2 weighted images.

\section{STATISTICS}

Age at onset distributions were compared by the Kruskal-Wallis test and averages by $t$ test. The relation between CMCT and height was 


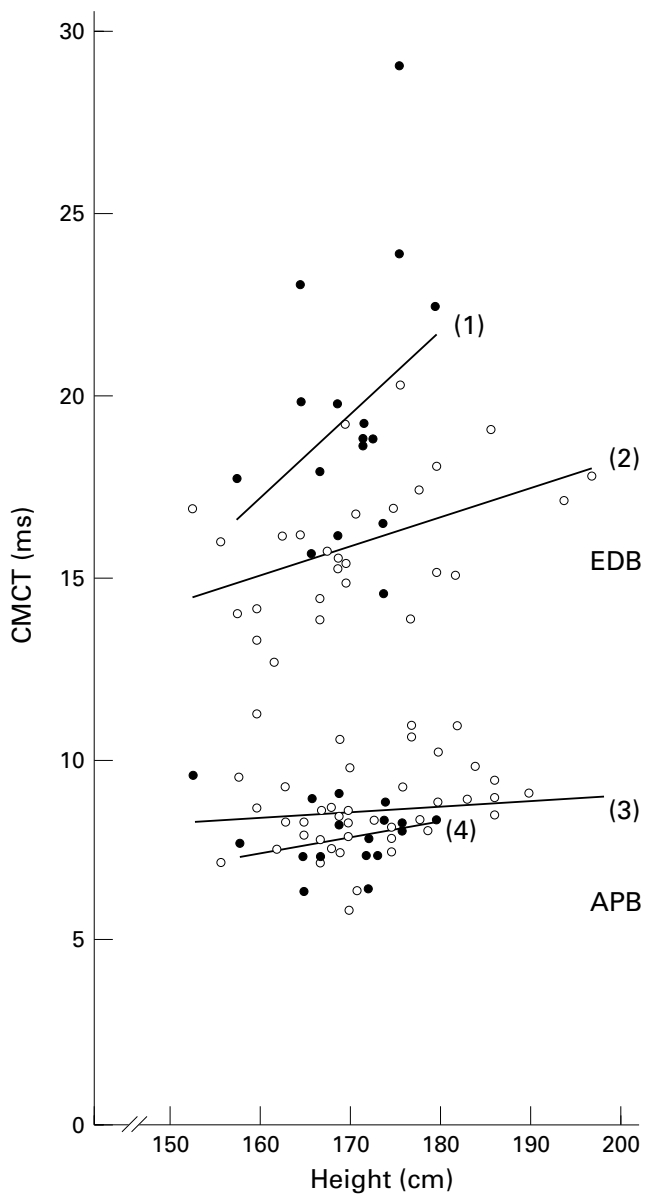

Figure 2 Central motor conduction time (CMCT) against height in 16 patients with ADPSP and in controls. $E D B=$ extensor digitorum brevis; $A P B=$ abductor pollicis brevis; filled circles = patients; open circles $=$ controls. The estimated regression lines (1) and (4) for the patients, (2) and (3) for the controls are shown:

(1) $C M C T=-19.46+0.23 \times$ height

(2) $C M C T=2.20+0.08 \times$ height, $(n=28)$;

(3) $C M C T=6.07+0.01 \times$ height, $(n=50)$;

(4) $C M C T=1.64+0.04 \times$ height. The regression coefficient 0.08 was significantly greater than $0(p=0.01)$.

examined by linear regression. Frequencies were compared by $\chi^{2}$ test. The significance level was taken as 0.05 .

\section{Results}

Autosomal dominant inheritance was apparent from the pedigrees (fig 1). Incomplete penetrance was not observed. At clinical examination of the 63 persons, 30 fulfilled the criteria of ADPSP index 3 (table 2). Twelve persons from families A, B, C, and D were scored 0-1, 1 , or 2 , nine of whom had no symptoms, but disclosed signs at examination. Eighteen persons were without any symptoms or signs of ADPSP (ADPSP index 0). In all the patients the onset was insidious and confined to the legs.

Table 2 shows the ages at onset in the five families. The age at onset varied both within and among families, but the five distributions of ages at onset did not differ significantly and we found no sex difference. We were not able to identify early or late onset families corresponding to the criteria proposed by Harding, ${ }^{18}$ except for family $\mathrm{B}$. which might be an early onset family. A feature not described before was found in the families A and E, in which five members had non-progressive childhood onset whereas the other affected family members had progressive adolescent or adult onset. Of 32 parent-offspring pairs in whom both a parent and a child were affected (ADPSP index 3 ), the onset of ADPSP occurred in an earlier decade of life in the child than in the parent in 24 pairs ("the anticipation group") and in the same decade or in a later decade in eight pairs $(p<0.01)$. To eliminate direct ascertainment bias, two parent-offspring pairs involving index patients were excluded. Of the remaining 30 pairs 22 were in the anticipation group and this was still a significant excess $(p<0.02)$, suggesting anticipation. The mean age at onset for the offspring at paternal transmission was 20.6 years and at maternal transmission 27.2 years but the difference was not significant. In all five families the median of the disability score was 2 which is in accordance with the fairly "benign" course of ADPSP. Two men, from families C (II-3) and E (II-1), with age at onset at 40 and 41 years are the only chairbound patients. In both cases the disease has been rapidly progressive with a duration of only nine and 12 years until they were immobilised.

Table 3 summarises the distributions of selected clinical features in the 30 patients with ADPSP index 3. All patients, except three with onset of symptoms from infancy, had normal motor milestones. The most frequent initial symptoms were stiffness in the legs $(93 \%)$, followed by rapid worn out shoes, especially outside in front $(87 \%)$, and gait unsteadiness $(87 \%)$. As a late symptom low backache was reported by $47 \%$ of the patients.

To test for an association between the duration of the disease and the occurrence of six clinical characteristics the patients with ADPSP index 3 were subdivided into two groups according to whether the duration of disease had been more or less than 10 years. Low backache occurred for relatively fewer patients if the duration of disease had been less than 10 years than if the duration had been more than 10 years ( 2 of $7=29 \% \quad v 12$ of $23=52 \%$ ). Also for upper limb hyperreflexia, weakness in the legs, decreased vibration sense, urinary symptoms, and nystagmus the percentages were lower for the group of patients with the short duration; however, probably due to the small groups none of the differences were significant.

Positive lod scores were obtained for the SPG4 markers on chromosome $2 p$ with a maximum multipoint lod score for the combined five families of $Z=8.60$ (data not shown).

Linkage analyses at the ADPSP loci on chromosomes $14 \mathrm{q}$ and $15 \mathrm{q}$ showed recombinations at each of them in all five families. Therefore the phenotype is unlikely to be caused by a mutation in a gene belonging to one of those two loci. Thus linkage analysis confined the ADPSP locus to chromosome 2p21-p24 (SPG4) in each of the five families.

At electrophysiological examination the SSEPs were normal at stimulation of the median nerves in 15 of 16 patients. One patient 
presented a slight prolongation of the central conduction time. At stimulation of the tibial nerves 11 of 16 were found normal. The abnormalities in the five patients were minor and insignificant, mostly confined to the lower limbs. The scatter diagram in figure 2 shows the CMCT in ms plotted against the height. For the extensor digitorum brevis the CMCT values tended to be higher for the patients than for the controls. For the controls the CMCT values increased on average with the height. A similar trend was seen for the patients, but the regression was not significant, possibly due to the small number. For the patients it also seemed that the points scattered to a greater extent around the regression line compared with the controls. We found no differences among the four families and no correlation between age at onset, duration of disease, any single sign, and the neurophysiological findings.

MRI disclosed mild spinal cord atrophy in two brothers from family C; in patient II- 1 at the level of Th 4-6 and in II-7 at the level of Th 3-7; however, this finding was reported by one radiologist only. Moderate cortical atrophy was found in one patient from family D (II-10) as well as in one control, both persons older than 50 years. White matter hyperintensity lesions in the cerebral hemispheres were seen in six patients from the four different families, compared with two persons in the 15 controls ( 6 of $16=38 \%$ v 2 of $15=13 \%$; the difference was not significant). Cerebellar or brainstem atrophy was not encountered.

\section{Discussion}

Linkage analyses confined the ADPSP locus in all five families to chromosome 2p21-p24; thus the families represent one and the same type of ADPSP.

Interfamilial and intrafamilial variation in age at onset was evident. The fluctuation within the families may reflect the fact that age at onset may be very difficult to date precisely, especially in ADPSP, a slowly progressive disorder, in which symptoms can go unnoticed for years as illustrated by the nine patients from among 12 with ADPSP index $0-1,1$, and 2 .

We did not find generation leaps. Incomplete penetrance was reported by Polo et $a l^{19}$ but as mentioned the high proportion of asymptomatic patients in ADPSP makes it risky to consider as unaffected an ancestor who has not been examined.

The phenomenon of anticipation may be a result of a recruitment bias favouring families in which the disease is detected earlier in children than in parents as proposed by Dürr et $a l^{11}$ or a biological phenomenon as indicated at least in the cases of "congenital" ADPSP seen in families $A$ and $E$. In other neurodegenerative autosomal dominantly inherited disorders such as Huntington's disease, ${ }^{20}$ dentatorubropallidoluysian atrophy, ${ }^{21}$ and Machado-Joseph disease $^{22}$ anticipation is well established and caused by futher elongation of the CAG repeat expansion in the respective genes.

Like Durr et $a l^{11}$ we found no parental bias but there was a tendency towards a lower age at onset in the offspring when the disease gene was transmitted paternally compared with maternal transmission, which is also described in the three neurodegenerative disorders mentioned above.

Phenotypic variability was evident, although the core features of ADPSP were homogenously expressed in all patients. However, low backache, not previously associated with ADPSP, was seen in all five families and probably this symptom may be a consequence of the laborious, lordotic gait of these patients. Dysdiadochokinesia, nystagmus, and dysarthria might be related to brainstem or cerebellar involvement; however, those features occurred in a minority of affected members only, and not in others from the same family and never in the probands. Therefore those families are still included in the category of pure ADPSP and multisystem involvement, as assessed by visual, brainstem, somatosensory evoked potentials, and studies of saccadic eye movements has been suggested by others also. ${ }^{11} 172324$

Probably due to differences in clinical criteria, in severity and duration of the disease, genetic heterogeneity, anatomical variation, and in recording techniques, neurophysiological studies of ADPSP have been rather inconsistent and conflicting. In our material SSEPs from the upper limbs were normal in 15 of 16 patients in accordance with the distributions of symptoms and signs of ADPSP and the findings of Bruyn et al. ${ }^{25}$ From 11 of 16 patients the SSEPs from the lower limbs were within the normal range whereas the abnormalities in five patients were only minor and insignificant, which is by contrast with the findings of Bruyn et al, who reported on abnormal tibial nerve SSEPs in 20 of 32 patients with ADPSP from nine kinships of unreported inheritance as opposed to one of 17 controls.

In our CMCT study, performed by transcranial magnetic stimulation, the MEPs from upper and lower limbs were all within the normal range, and no significant difference was found, compared with controls, between or within the families. However, there was a tendency for delay of the MEPs in the lower limbs. In a study by Claus et al four patients with ADPSP were examined with recordings from both upper and lower limbs. They had normal values for the upper limb recordings in three patients but abnormal values for the lower limbs in all four patients. A major problem in most previous studies, however, is whether the transcranial magnetic stimulation is applied with an intensity sufficient to evoke an MEP. Delayed or absent MEPs may be seen in patients with an increased motor threshold. In our study we therefore applied transcranial magnetic stimulation with an intensity related to motor threshold and our results indicate that conduction in the central motor pathways in most cases is normal and that demyelination of the first motor neuron is not a major pathophysiological phenomenon. Therefore, we suggest that a local spinal mechanism may also be involved in the pathogenesis of the spasticity. 
In the MRI study thoracic spinal cord atrophy was described in two brothers but only by one radiologist. Thus it seems that atrophy of the spinal cord may be difficult to assess and presumably a very late finding as evaluated qualitatively by MRI. Also an MRI study of patients from a family with $\mathrm{X}$ linked pure hereditary spastic paraparesis disclosed a normal spinal cord in the only patient who had a spinal cord MRI performed. ${ }^{26}$ MRI of the cerebrum, cerebellum, and brainstem was mostly normal in our patients. Ormerod et $a l^{10}$ reported on seven patients with autosomal dominant hereditary spastic paraparesis either in the pure form (five cases) or with additional features who had MRI of the brain performed. One patient with the pure form had white matter hyperintensity lesions whereas the MRI was normal in the four other patients with ADPSP. We found white matter hyperintensity lesions in the six oldest patients with ADPSP compared with two controls in accordance with the finding of an almost linear increase in the number of volunteers with white matter hyperintensity lesions with aging for men and women in a MRI study of 142 healthy people by Christiansen et al $^{27}$

In conclusion, the clinical core features of ADPSP linked to chromosome 2p21-p24 were homogeneously expressed in all patients but some symptoms and signs were only found in some families and only in a few patients from those families, a feature which in part may account for the varying definition of ADPSP from study to study. Thus it seems that ADPSP may be more or less "pure" and that "pure plus" families may exist without being "complicated", presumably depending both on the specific mutation in the family and on unknown modulating genetic and environmental factors as well. The anticipation, the varying age at onset, and the phenotypic heterogeneity might suggest an unstable trinucleotide repeat as the underlying molecular mutation, ${ }^{28}$ but further investigations, including molecular genetic studies, will be required to identify the genes responsible of ADPSP to elucidate the pathophysiological processes and to settle the issue of classification.

Financial support was obtained from the Danish Medical Association Research Fund; the Danish Hospital Foundation for Medical Research, Region of Copenhagen, The Faroe Islands, and Greenland; the Signe and Peter Gregersen Fund the Lily Benthine Lund Fund; and the Dr Eilif Trier-Hansen and wife Ane Trier-Hansen Fund is gratefully acknowledged. This study would not have been possible without the participation of patients with ADPSP and their families.

1 Harding AE. Classification of the hereditary ataxias and paraplegias. Lancet 1983;1:1151-5.

2 Hazan J, Lamy C, Melki J, Munnich A, Recondo J, Weissen-

bach J. Autosomal dominant familial spastic paraplegia is genetically heterogeneous and one locus maps to chromoome 14q. Nat Genet 1993;5:163-7.

3 Hazan J, Fontaine B, Bruyn RPM, et al. Linkage of a new ocus for autosomal dominant familial spastic paraplegia to chromosome 2p. Hum Mol Genet 1994;3:1569-73.

4 Fink JK, Wu CB, Jones SM, et al. Autosomal dominant familial spastic paraplegia: tight linkage to chromosome 15q. Am 尹 Hum Genet 1995;56:188-92.

5 Fink JK, Heiman-Patterson T, for the Hereditary Spastic Paraplegia Working Group. Hereditary spastic paraplegia: advances in genetic research. Neurology 1996;46:1507-14.

6 Bruyn RPM. The neuropathology of hereditary spastic Bruyn RPM. The neuropathology of hereditary spastic
paraparesis. Clin Neurol Neurosurg 1992;94(suppl):S16-8.

paraparesis. Clin Neurol Neurosurg 1992;94(suppl):S16-8.
7 Claus D, Waddy HM, Harding AE, Murray NMF, Thomas Claus D, Waddy HM, Harding AE, Murray NMF, Thomas
PK. Hereditary motor and sensory neuropathies and PK. Hereditary motor and sensory neuropathies and Ann Neurol 1990;28:43-9.

8 Schady W. Dick JPR, Sheard A, Crampton S. Central motor conduction studies in hereditary spastic paraplegia. $\mathcal{F} \mathrm{Neu}$ rol Neurosurg Psychiatry 1991;54:775-9.

9 Pelosi L, Lanzillo B. Perretti A, Santoro L, Blumhardt L, Caruso G. Motor and somatosensory evoked potentials in hereditary spastic paraplegia. F Neurol Neurosurg Psychiatry 1991;54:1099-102.

10 Ormerod IEC, Harding AE, Miller DH, et al. Magnetic resonance imaging in degenerative ataxic disorders. $\mathcal{F} \mathrm{Neu}$ rol Neurosurg Psychiatry 1994;57:51-7.

11 Dürr A, Brice A, Serdaru M, et al. The phenotype of "pure" autosomal dominant spastic paraplegia. Neurology 1994;44: $1274-7$

12 Bruyn RPM, Scheltens PH. Hereditary spastic paraparesis (Strumpell-Lorrain). In: Vinken PJ, Bruyn GW, eds. Handbook of clinical neurology (2nd series). Amsterdam: North Holland Publishing, 1991;15/59:301-18.

13 Behan WMH, Maia M. Strümpell's familial spastic paraplegia: genetics and neuropathology. I Neurol Neurosurg Psychiatry 1974;37:8-20.

14 Bürger J, Metzke H, Paternotte C, Schilling F, Hazan J, Reis A. Autosomal dominant spastic paraplegia with anticipation maps to a 4-cM interval on chromosome $2 \mathrm{p} 21 \mathrm{p} 24$ in a large German family. Hum Genet 1996;98:371-5.

15 Schäffer AA, Gupta SK, Shriram K, Cottingham RW. Avoiding recomputation in linkage analysis. Hum Hered 1994:44:225-37.

16 Dib C, Fauré S, Fizames C, et al. The Généthon human genetic linkage map. Nature 1996;380:152-4.

17 Pedersen L, Trojaborg W. Visual, auditory and somatosensory pathway involvement in hereditary cerebellar ataxia, Friedreich's ataxia and familial spastic paraplegia. Electrenceph Clin Neurophysiol 1981;52:283-97.

18 Harding AE. Herditary "pure" spastic paraplegia: a clinical and genetic study of 22 families. F Neurol Neurosurg Psychiatry 1981;44:871 -83.

19 Polo JM, Calleja J. Combarros O. Berciano J. Hereditary pure" spastic paraplegia: a study of nine families. $\mathcal{F}$ Neurol Neurosurg Psychiatry 1993;56:175-81.

20 Nørremølle A, Sørensen SA, Fenger K, Hasholt L. Correlation between magnitude of CAG repeat length alterations and length of the paternal repeat in paternally inherited Huntington's disease. Clin Genet 1995;47:113-7.

21 Nielsen JE, Sørensen SA, Hasholt L, Nørremølle A. Dentatorubral-pallidoluysian atrophy. Clinical features of a Dentatorubral-pallidoluysian atrophy. Clinical features of a
five-generation Danish family. Mov Disord 1996;11:53341 .

22 Maciel P, Gaspar C, DeStefano AL, et al. Correlation between CAG repeat length and clinical features in Machado-Joseph disease. Am F Hum Genet 1995;57:54-61 .

23 Schady W. Sheard A. A quantitative study of sensory function in hereditary spastic paraplegia. Brain 1990;113: $709-20$

24 Tedeschi G, Allocca S, Di Costanzo A, et al. Multisystem involvement of the central nervous system in Strümpell's disease. A neurophysiological and neuropsychological study. $\mathcal{F}$ Neurol Sci 1991;103:55-60.

25 Bruyn RPM, van Dijk JG, Scheltens P, Boezeman EHJF, Ongerboer de Visser BW. Clinically silent dysfunction of dorsal columns and dorsal spinocerebellar tracts in hereditary spastic paraparesis. F Neurol Sci 1994;125:206-11.

26 Cambi F, Tartaglino L, Lublin F, McCarren D. X-linked pure familial spastic paraparesis. Characterization of a large pure familial spastic paraparesis. Characterization of a large
kindred with magnetic resonance imaging studies. Arch Neurol 1995;52:665-9.

27 Christiansen P, Larsson HBW, Thomsen C, Wieslander SB, Henriksen O. Age dependent white matter lesions and brain volume changes in healthy volunteers. Acta Radiol 1994;35:117-22.

28 Monckton DG, Caskey CT. Unstable triplet repeat diseases. Circulation 1995;91:513-20. 